\title{
Clinical Performance of SARS-CoV-2 IgG and IgM Tests Using an Automated Chemiluminescent Assay
}

\author{
Jin-an ZHOU ${ }^{1}$, Hao-long ZENG ${ }^{2}$, Ling-yan DENG ${ }^{2}$, Hui-jun $\mathrm{LI}^{2 \#}$ \\ ${ }^{I}$ Department of Blood Transfusion, Tongji Hospital, Tongji Medical College, Huazhong University of Science and Technology, \\ Wuhan 430030, China \\ ${ }^{2}$ Department of Laboratory Medicine, Tongji Hospital, Tongji Medical College, Huazhong University of Science and Technology, \\ Wuhan 430030, China
}

(C) Huazhong University of Science and Technology 2021

\begin{abstract}
Summary: Serology tests for viral antibodies provide an important tool to support nucleic acid testing for diagnosis of the novel coronavirus disease 2019 (COVID-19) and is useful for documenting previous exposures to SARS-CoV-2, the etiological agent of COVID-19. The sensitivities of the chemiluminescent SARS-CoV-2 IgG/IgM immunoassay were assessed by using serum samples collected from 728 patients testing positive for SARS-CoV-2 RNA. The specificity was evaluated on a panel of 60 serum samples from non-COVID-19 patients with high levels of rheumatoid factor, antinuclear antibody, or antibodies against Epstein-Barr virus (EBV), cytomegalovirus (CMV), mycoplasma pneumonia, human respiratory syncytial virus (RSV), adenovirus, influenza A or influenza B. The imprecision and interference were assessed by adopting the Clinical and Laboratory Standards Institute (CLSI) EP15-A2 and EP7-A2, respectively. Sensitivities between 1 and 65 days after onset of symptoms were $94.4 \%$ and $78.7 \%$, for IgG and IgM test, respectively. The sensitivity increased with the time after symptom onset, and rose to the top on the 22nd to 28th days. The total imprecision (CVs) was less than $6.0 \%$ for $\operatorname{IgG}$ and less than $6.5 \%$ for IgM. Limited cross-reactions with antibodies against EBV, CMV, mycoplasma pneumonia, human RSV, adenovirus, influenza A or influenza B were found. These data suggested the chemiluminescent SARS-CoV-2 IgG and IgM, assay with reliable utility and sensitivity, could be used for rapid screening and retrospective surveillance of COVID-19.
\end{abstract}

Key words: COVID-19; SARS-CoV-2; IgG; IgM; chemiluminescence

It has been reported that, of the total number of patients confirmed with coronavirus disease 2019 (COVID-19), more than $80 \%$ were mild cases, with older adults and people who have other conditions being most at risk ${ }^{[1]}$. This made the retrospective surveillance an important means to screen for severe acute respiratory syndrome (SARS)-associated coronavirus $(\mathrm{CoV})-2$ infection and interrupt undetected chains of disease transmission, as such surveillance may be key to tracking those mild cases ${ }^{[2]}$. The current laboratory method for identifying COVID-19 is mainly nucleic acid RT-PCR test, which is believed to be the most sensitive method for early identification of infection ${ }^{[3]}$. However, the RT-PCR test may not be an ideal tool for using in retrospective mass screening, as viral load rapidly declines 9 or 10 days after disease onset ${ }^{[4,5]}$.

Jin-an ZHOU, E-mail: 2001tj0603@tjh.tjmu.edu.cn \#Corresponding author, E-mail: lihuijun@tjh.tjmu.edu.cn *The study was supported by the National Natural Science Foundation of China (No. 31600666).
More importantly, quite a number of false negatives occurred due to the variations during throat swab collection. The long turnaround times for RT-PCR of SARS-CoV-2 should also be considered ${ }^{[6]}$. Therefore, development and evaluation of new tests for specific antibodies of SARS-CoV-2 in patient blood are in urgent need.

It is widely accepted that IgM provides the first line of defense during viral infections, prior to generation of adaptive, high affinity $\operatorname{IgG}$ responses that are important for long-term immunity and immunological memory ${ }^{[7,8]}$. It was reported that after SARS-CoV infection, $\operatorname{IgM}$ antibody could be detected in patient blood after 3-6 days and IgG could be detected after 8 days $^{[9]}$. While for Middle East respiratory syndrome coronavirus (MERS) infection, IgG and IgM tests showed optimal sensitivity after 21 days post onset of illness ${ }^{[10]}$. In the present study, we aimed to investigate the performance of SARS-CoV-2 IgG and IgM test using a new automated chemiluminescence assay on well-characterized samples from laboratory-confirmed 
COVID-19 patients and to challenge the test against a panel of samples known to cause possible crossreactions.

\section{MATERIALS AND METHODS}

\subsection{Samples and Laboratory Diagnosis}

A total of 728 serum samples from 728 COVID-19 patients were collected to estimate the diagnostic accuracy of the SARS-CoV-2 antibody test. These patients were all residents of Wuhan, China, and admitted to the Tongji Hospital of Tongji Medical College, Huazhong University of Science and Technology in Wuhan of China, from January 27 to March 2, 2020, and confirmed to have COVID-19 according to the Guidelines of the Diagnosis and Treatment of New Coronavirus Pneumonia published by the National Health Commission of China ${ }^{[11]}$. Of these patients, $320(44.0 \%)$ were men and the median age was 63 years (range: 15-92).

A second panel consisted of 60 serum samples were used to assess the degree of possible crossreactions. These samples were from patients with high levels of rheumatoid factor (RF) (range: $>1500$ $\mathrm{IU} / \mathrm{mL}, \quad n=2 ; \leq 1500 \quad \mathrm{IU} / \mathrm{mL}, \quad n=5)$, antinuclear antibody (ANA) $(1: 320-1: 3200, n=14)$, or IgM and IgG antibodies against Epstein-Barr virus (EBV) $(n=10)$, cytomegalovirus (CMV) $(n=3)$, mycoplasma pneumonia $(n=5)$, human respiratory syncytial virus (RSV) $(n=2)$, adenovirus $(n=2)$, influenza A $(n=11)$ or influenza B virus $(n=6)$.

The study was conducted in accordance with guidelines approved by the Ethics Committees of Tongji Hospital (China) (ID:TJ-IRB20200220). Oral informed consents were obtained from all the patients or patients' family members.

\subsection{Laboratory Measurements}

SARS-CoV-2 antibodies (IgG, IgM) were determined by using a fully-automated, two-step chemilumi-nescent immunoassay kit (iFlash SARSCoV-2 IgG/IgM, YHLO Biotech. Shenzhen, China), with the use of specific recombinant proteins (nucleocapsid protein and spike protein of the SARSCoV-2). Paramagnetic carboxylated-microparticles were coated with the recombinant proteins through cross-linking by N-ethyl-N'-(3-dimethylaminopropyl) carbodiimide. Mouse monoclonal antihuman IgM or IgG was conjugated with NSP-DMAE-NHS. In the first step, sample, specimen treating agent (for IgM tests, sample dilution is needed), and recombinant SARSCov-2 antigen-coated paramagnetic microparticles were combined. The reaction mixture was washed and anti-human acridinium-labeled conjugate was added to form a reaction complex in the second step. Following another wash cycle, pre-trigger and trigger solutions were added to the reaction mixture. The final chemiluminescent reaction was expressed as relative light units (RLUs). The concentration of SARS-CoV-2 IgM or IgG in the sample was determined by comparing the RLU of a sample to the RLU determined from two calibrators. The manufacturer's suggested cutoff for SARS-CoV-2 IgG and IgM was $>10 \mathrm{AU} / \mathrm{mL}$. The repeatability and reproducibility was $\leq 10 \%$ and $\leq 15 \%$, respectively.

\subsection{Imprecision}

Imprecision was verified over five consecutive days using two levels of pooled human serum samples, tested in triplicate in accordance with Clinical and Laboratory Standards Institute (CLSI) document EP15-A2 ${ }^{[12]}$. Two fresh serum pools indicated two different concentration levels, were prepared, split to aliquots, stored at $-70^{\circ} \mathrm{C}$, and then thawed immediately in the $37^{\circ} \mathrm{C}$ water until measurement. During the imprecision verification, reagent of the same lot number was used by one operator.

\subsection{Interference}

Interference testing of hemoglobin, bilirubin and lipaemia was performed based on CLSI document EP7-A2 ${ }^{[13]}$. Two base serum samples with two different concentrations of SARS-CoV-2 IgG/IgM were prepared. The Sysmex Interference Check.A Plus kit (Sysmex, Japan) was used to test the potential interferences of hemoglobin, bilirubin and lipaemia as previously reported ${ }^{[14]}$.

\subsection{Statistical Analysis}

The SPSS version 22.0 (SPSS, USA) was used as statistical package to estimate sensitivity and specificity with $95 \%$ confidence intervals. The crossreactivity study was treated as a descriptive analysis due to the insufficient sample size and therefore no statistical analyses were conducted.

\section{RESULTS}

\subsection{Diagnostic Sensitivity}

Of the 728 laboratory-confirmed COVID-19 patients, 687 (94.4\%) were positive for the SARSCoV-2 IgG test, and $573(78.7 \%)$ were positive for IgM test. The diagnostic sensitivity to detect IgG and IgM antibodies per time period of onset of symptoms is listed in table 1. From 1 to 4 days post-symptom onset, four patients were confirmed with COVID-19, and all showed negative for both the SARS-CoV-2 IgG and IgM test. From 5 to 14 days post-symptom onset, 35/53 $(66.0 \%)$ and 25/53 (47.2\%) laboratory-confirmed patients were positive, respectively, for $\operatorname{IgG}$ test and IgM test. From 15 to 35 days post-symptom onset, $523 / 540(96.9 \%)$ and 452/540 (83.7\%) patients were positive, respectively, for IgG test and IgM test. And after 36 days post-symptom onset, 129/131 (98.5\%) and 96/131 (73.3\%) patients were positive, respectively, for $\mathrm{IgG}$ test and IgM test. The variation curve of sensitivity 
Table 1 Sensitivtiy of the IgG and IgM test to detect IgG and IgM antibodies per time period in samples from patients with a confirmed SARS-COV-2 infection

\begin{tabular}{|c|c|c|c|c|c|c|c|}
\hline \multirow{2}{*}{ Days post-symptom onset } & \multirow{2}{*}{$n$} & \multirow{2}{*}{ Range } & \multirow{2}{*}{ Median } & \multicolumn{2}{|c|}{ IgG test } & \multicolumn{2}{|c|}{ IgM test } \\
\hline & & & & Positive ratio & Sensitivity $(95 \% \mathrm{CI})$ & Positive ratio & Sensitivity $(95 \% \mathrm{CI})$ \\
\hline$\leq 4$ & 4 & $2-4$ & 3 & NA & NA & NA & NA \\
\hline $5-14$ & 53 & $5-14$ & 11 & $35 / 53$ & $0.66(0.53-0.79)$ & $25 / 53$ & $0.47(0.33-0.61)$ \\
\hline $15-35$ & 540 & $15-35$ & 26 & $523 / 540$ & $0.97(0.95-0.98)$ & $452 / 540$ & $0.84(0.81-0.87)$ \\
\hline$\geqq 36$ & 131 & $36-65$ & 39 & $129 / 131$ & $0.98(0.96-1.00)$ & $96 / 131$ & $0.73(0.66-0.81)$ \\
\hline
\end{tabular}

$\mathrm{NA}=$ not applicable

for SARS-CoV-2 IgG and IgM test according to time period of post-symptom onset is shown in fig. 1. The positive rates for both the IgG and IgM test could be found to increase with the time after symptom onset, and both rose to the top on the 22nd to 28th days. From then on, the $\operatorname{IgG}$ test kept a high positive rate of more than $95 \%$, while IgM test declined to lower than $75 \%$. These results demonstrated diagnostic sensitivity of the IgG test was better than the IgM test for detecting the SARS-CoV-2 antibodies in our assay, and both of the tests showed positive rates changed with the days after onset of symptoms in COVID-19 patients.

\subsection{Cross-reactivity}

A total of 60 serum samples from patients with

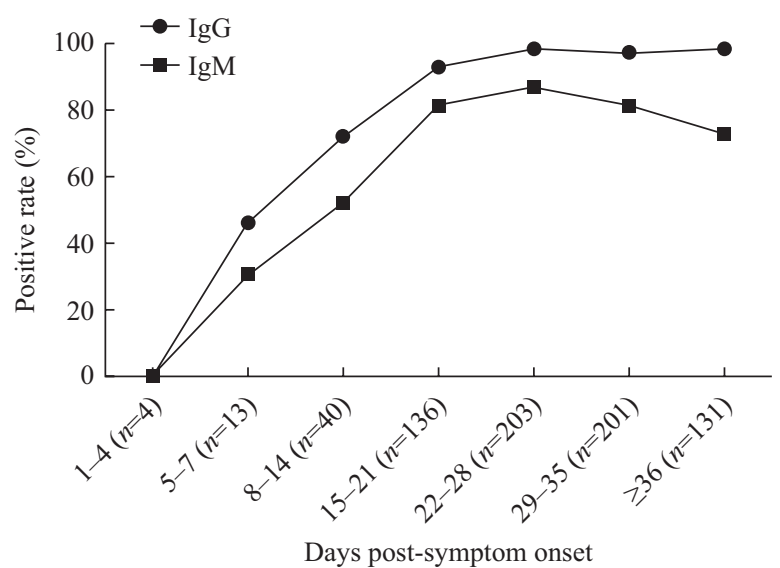

Fig. 1 Positive rate of SARS-CoV-2 IgG and IgM tests in relation to the days after symptom onset in 724 confirmed COVID-19 patients high levels of RF, ANA or IgM and IgG antibodies against EBV, CMV, mycoplasma pneumoniae, human RSV, adenovirus, influenza A or influenza B virus, were tested. The results of cross-reactivity estimation for SARS-CoV-2 IgG assay and IgM assay are shown in table 2. The positive $\operatorname{IgG}$ and $\operatorname{IgM}$ was found in $1 / 2$ samples with RF $(>1500 \mathrm{IU} / \mathrm{mL})$. The positive IgM was also found in $1 / 5$ samples with mycoplasma pneumonia. No other cross-reactivity was found.

\subsection{Interference}

Interferences of hemoglobin (up to $5 \mathrm{~g} / \mathrm{L}$ ), unconjugated bilirubin (up to $20 \mathrm{mg} / \mathrm{dL}$ ) and lipaemia (up to 1410 FTU) were estimated and could be accepted with changes less than or around $10 \%$. As listed in table 3, we found at the low level (Level 1) of the SARS-CoV-2 antibodies, result of the IgG test was interfered by hemoglobin (up to $500 \mathrm{mg} / \mathrm{dL}$ ) with the bias of $10.86 \%$, while the IgM test was interfered by bilirubin (conjugated) (up to $20 \mathrm{mg} / \mathrm{dL}$ ) with the bias of $-12.18 \%$.

\subsection{Imprecision}

Determination of SARS-CoV-2 IgG and IgM antibodies was very precise, and within the range of repeatability and reproducibility reported by the manufacturer (table 4).

\section{DISCUSSION}

In the present study, we assessed the diagnostic performance for SARS-CoV-2 IgG and IgM test using a new automated chemiluminescent assay. Serum

Table 2 Possible cross-reactivity estimated for SARS-CoV-2 IgG assay and IgM assay

\begin{tabular}{|c|c|c|c|c|c|}
\hline \multirow{2}{*}{ Possible cross-reaction } & \multirow{2}{*}{$\mathrm{N}$} & \multicolumn{2}{|c|}{ SARS-CoV-2 IgG positive } & \multicolumn{2}{|c|}{ SARS-CoV-2 IgM positive } \\
\hline & & $n$ & Value (AU/mL) & $n$ & Value $(\mathrm{AU} / \mathrm{mL})$ \\
\hline EBV (IgG+, IgM+) & 10 & 0 & NA & 0 & NA \\
\hline $\mathrm{CMV}(\operatorname{IgG}+, \operatorname{IgM}+)$ & 3 & 0 & NA & 0 & NA \\
\hline Mycoplasma pneumoniae ( $\operatorname{IgG}+, \operatorname{IgM}+)$ & 5 & 0 & NA & 1 & 10.45 \\
\hline Human respiratory syncytial virus $(\operatorname{IgG}+, \operatorname{IgM}+)$ & 2 & 0 & NA & 0 & NA \\
\hline Adenovirus (IgG+, IgM) & 2 & 0 & NA & 0 & NA \\
\hline Influenza A virus ( $\operatorname{IgG}+, \operatorname{IgM}+$ ) & 11 & 0 & NA & 0 & NA \\
\hline Influenza B virus ( $\operatorname{IgG}+$, IgM + ) & 6 & 0 & NA & 0 & NA \\
\hline $\mathrm{RF}(<1500 \mathrm{IU} / \mathrm{ml})$ & 5 & 0 & NA & 0 & NA \\
\hline $\mathrm{RF}(>1500 \mathrm{IU} / \mathrm{ml})$ & 2 & 1 & 16.47 & 1 & 13.05 \\
\hline ANA $(1: 320-1: 3200)$ & 14 & 0 & NA & 0 & NA \\
\hline
\end{tabular}

NA: not applicable; RF, rheumatoid factor; ANA, antinuclear antibody; EBV: Epstein-Barr virus; CMV: cytomegalovirus 
Table 3 Interference estimated for SARS-CoV-2 IgG and IgM tests

\begin{tabular}{|c|c|c|c|c|c|c|c|}
\hline \multirow[t]{2}{*}{ Interference } & \multirow{2}{*}{$\begin{array}{l}\text { Max concentration } \\
\text { of interference }\end{array}$} & $\begin{array}{c}\text { Control } \\
(\mathrm{AU} / \mathrm{mL})\end{array}$ & $\begin{array}{c}\text { Added interference } \\
(\mathrm{AU} / \mathrm{mL})\end{array}$ & $\begin{array}{c}\text { Changes } \\
(\%)\end{array}$ & $\begin{array}{c}\text { Control } \\
(\mathrm{AU} / \mathrm{mL})\end{array}$ & $\begin{array}{c}\text { Added interference } \\
(\mathrm{AU} / \mathrm{mL})\end{array}$ & $\begin{array}{c}\text { Changes } \\
(\%)\end{array}$ \\
\hline & & \multicolumn{3}{|c|}{ SARS-CoV-2 IgG level 1} & \multicolumn{3}{|c|}{ SARS-CoV-2 IgG level 2} \\
\hline Bilirubin (conjugated) & $20 \mathrm{mg} / \mathrm{dL}$ & 8.24 & 8.66 & 5.10 & 58.26 & 58.48 & 0.38 \\
\hline Bilirubin (unconjugated) & $20 \mathrm{mg} / \mathrm{dL}$ & 9.46 & 8.56 & -9.51 & 56.94 & 58.99 & 3.60 \\
\hline Hemoglobin & $500 \mathrm{mg} / \mathrm{dL}$ & 8.01 & 8.88 & 10.86 & 56.72 & 56.68 & -0.07 \\
\hline \multirow[t]{2}{*}{ Lipaemia } & 1410 FTU & 7.96 & 8.45 & 6.16 & 57.84 & 57.17 & -1.16 \\
\hline & & \multicolumn{3}{|c|}{ SARS-CoV-2 IgM level 1} & \multicolumn{3}{|c|}{ SARS-CoV-2 IgM level 2} \\
\hline Bilirubin (conjugated) & $20 \mathrm{mg} / \mathrm{dL}$ & 8.7 & 7.64 & -12.18 & 40.11 & 40.14 & 0.07 \\
\hline Bilirubin (unconjugated) & $20 \mathrm{mg} / \mathrm{dL}$ & 8.45 & 8.66 & 2.49 & 38.88 & 40.41 & 3.94 \\
\hline Hemoglobin & $500 \mathrm{mg} / \mathrm{dL}$ & 7.27 & 7.88 & 8.39 & 37.92 & 38.70 & 2.06 \\
\hline Lipaemia & 1410 FTU & 7.89 & 7.81 & -1.01 & 38.96 & 40.10 & 2.93 \\
\hline
\end{tabular}

Table 4 Imprecision estimated for SARS-CoV-2 IgG and IgM tests

\begin{tabular}{|c|c|c|c|c|c|c|c|}
\hline \multirow{2}{*}{ Serum pools } & \multirow{2}{*}{ Mean } & \multicolumn{2}{|c|}{ Within-run } & \multicolumn{2}{|c|}{ Between-run } & \multicolumn{2}{|c|}{ Total } \\
\hline & & S.D. & CV $(\%)$ & S.D. & CV $(\%)$ & S.D. & CV (\%) \\
\hline IgG level 1 & $8.75 \mathrm{AU} / \mathrm{mL}$ & 0.14 & 1.66 & 0.48 & 5.45 & 0.50 & 5.70 \\
\hline IgG level 2 & $59.45 \mathrm{AU} / \mathrm{mL}$ & 1.56 & 2.62 & 0.97 & 1.64 & 1.84 & 3.09 \\
\hline IgM level 1 & $3.85 \mathrm{AU} / \mathrm{mL}$ & 0.22 & 5.75 & 0.08 & 2.08 & 0.24 & 6.11 \\
\hline IgM level 2 & $45.41 \mathrm{AU} / \mathrm{mL}$ & 2.24 & 4.94 & 1.54 & 3.38 & 2.72 & 5.99 \\
\hline
\end{tabular}

samples from 728 patients with laboratory-confirmed COVID-19 were used, of which the time post symptom onset was from 1 to 65 days. Our results revealed a positive rate of $94.4 \%$ for $\mathrm{IgG}$ test and $78.7 \%$ for IgM test to determine SARS-CoV-2 antibodies. A IgG-IgM combined antibody test for SARS-CoV-2 infection diagnosis developed recently by Yi et al showed an overall testing sensitivity of $88.66 \%{ }^{[6]}$, which was nearly equivalent to our estimation data.

Seroconversion for $\operatorname{IgG}$ and IgM occurred nearly simultaneously in patients infected with SARS$\mathrm{CoV}^{[15]}$, but the IgM against SARS-CoV-2 in this study showed a lower positive rate than IgG. This difference of sensitivities between IgG and IgM detection was also reported by a previous study of SARS-CoV ${ }^{[16]}$. We speculated that the difference could be due to the concentration of IgM was lower than that of IgG in blood after SARS-CoV-2 infection, also possibly due to the different capture sensitivity between the $\operatorname{IgG}$ and IgM regents in our assay. Moreover, previous studies of SARS-CoV indicated that the IgG and IgM increased to detectable levels at the second or third week after onset of symptoms ${ }^{[16,17]}$. In our study, both the IgG and IgM against SARS-CoV-2 could be detected as early as on the 5th to 7 th days post onset of illness, with the positive rate of $46.2 \%$ and $30.8 \%$, respectively. The positive rate of the two antibodies rose in the following days and reached the peak levels on the 22nd to 28th days post onset of symptoms. These data suggested the detection sensitivity seemed to be correlated with the concentrations of the antibodies in blood. As we know, IgM appears mainly in the early stage of infection. The SARS-CoV IgM antibody was reported to be decreased on the 28th day and dropped to below a detectable level on about the 90th day post onset of illnes ${ }^{[8]}$. Our data showed the IgM positive rate of SARS-CoV-2 was also decreased 28 days after onset of symptoms, which seems to be consistent with the variations of antibody titers.

Many serological tests have been developed for the diagnosis of virus infection, but false-positive reactions in negative serum may occur because of the immunological cross-reactivity with other anti-virus antibodies $^{[2,9]}$. We challenged the test with samples containing potential cross- reacting antibodies. No obvious cross-reactions were found with the other common pathogens including EBV, CMV, mycoplasma pneumonia, human RSV, adenovirus, and influenza A or influenza $B$ virus, and with autoimmune diseases like ANA. However, cross-reaction was found in samples with high concentration of RF $(>1500 \mathrm{IU} / \mathrm{mL})$ for both SARS-CoV-2 IgG and IgM tests. Hence, interference of RF should be taken into account for patients with high concentration of RF. Additionally, since lipemia, hemolysis, and icterus commonly interfere with laboratory tests that use optical methods ${ }^{[18]}$, and considering the automated chemiluminescent assay used in the present study to determine the SARS-CoV-2 antibodies, we further estimated the interferences of these factors based on Clinical \& Laboratory Standards Institute (CLSI) Interference Testing in Clinical Chemistry; Approved Guideline-Second Edition (EP7-A2), and found hemolysis and icterus may interfere with the test results when the antibody titer was low. The imprecision estimated according to that CLSI EP15-A2 is all less than $10 \%$ for withinrun and between run. All these results suggested the automated chemiluminescent assay in this study could be a reliable test for determination of SARS-CoV-2 IgG and IgM. 
This study has some limitations. The patient cohort used mainly consisted of COVID-19 patients living in Wuhan, and the number of patients infected with other pathogens or with autoimmune diseases was small. Further assessments of the test are needed for samples from COVID-19 patients of other endemic regions. In addition, we did not have the opportunity to include samples from patients of more than 65 days post symptom onset. Therefore, further studies are needed to estimate the sensitivity in samples beyond this date.

In conclusion, The IgG and IgM test for determination of SARS-CoV-2 antibodies, using an automated chemiluminescent assay, showed high sensitivities even in early samples in combination with high precision, limited cross-reaction and interference. The data makes the IgG and IgM test a reliable test in surveillance for SARS-CoV-2 infection and may be key to interrupt undetected chains of disease transmission.

\section{Conflict of Interest Statement}

The authors declare that they have no conflict of interest.

\section{REFERENCES}

1 Novel Coronavirus Pneumonia Emergency Response Epidemiology Tean, The epidemiological characteristics of an outbreak of 2019 novel coronavirus diseases (COVID-19) in China. Zhonghua Liu Xing Bing Xue Za Zhi, 2020,41:145-151

2 Hsueh PR, Kao CL, Lee CN, et al, SARS antibody test for serosurveillance. Emerg Infect Dis, 2004,10(9):15581562

3 Jin $\mathrm{YH}$, Cai L, Cheng ZS, et al, A rapid advice guideline for the diagnosis and treatment of 2019 novel coronavirus (2019-nCoV) infected pneumonia (standard version). Mil Med Res, 2020,7(1):4

4 Grant PR, Garson JA, Tedder RS, et al. Detection of SARS coronavirus in plasma by real-time RT-PCR. N Engl J Med, 2003,349(25):2468-2469

5 Peiris JS, Chu CM, Cheng VC, et al. Clinical progression and viral load in a community outbreak of coronavirusassociated SARS pneumonia: a prospective study. Lancet, 2003,361(9371):1767-1772

6 Huang C, Wang Y, Li X, et al. Clinical features of patients infected with 2019 novel coronavirus in Wuhan, China. Lancet, 2020, 395(10223):497-506
7 Racine R, Winslow GM. IgM in microbial infections: taken for granted? Immunol lett, 2009,125(2):79-85

8 Li G, Chen X, Xu A. Profile of specific antibodies to the SARS-associated coronavirus. N Engl J Med, 2003,349(5):508-509

9 Lee HK, Lee $\mathrm{BH}$, Seok $\mathrm{SH}$, et al. Production of specific antibodies against SARS-coronavirus nucleocapsid protein without cross reactivity with human coronaviruses $229 \mathrm{E}$ and OC43. J Vet Sci, 2010,11(2):165-167

10 Ko JH, Muller MA, Seok H, et al. Suggested new breakpoints of anti-MERS-CoV antibody ELISA titers: performance analysis of serologic tests. Eur J Clin Microbiol Infect Dis, 2017,36(11):2179-2186

11 NHCo C. Diagnosis and Treatment Scheme of New Coronavirus Infected Pneumonia. 2020.

12 Institute, CaLS, User Verification of Performance for Precision and Trueness; Approved Guideline, second edition. Clinical and Laboratory Standards Institute, Wayne, PA, USA 2006.

13 Institute, CaLS, Interference Testing in Clinical Chemistry; Approved Guideline-Second Edition. EP7-A2, Clinical and Laboratory Standards Institute, Wayne, PA 2005.

14 Deng L, Xiong Z, Li H, et al. Analytical validation and investigation on reference intervals of aldosterone and renin in Chinese Han population by using fully automated chemiluminescence immunoassays. Clin Biochem, 2018,56,89-94

15 Hsueh PR, Huang LM, Chen PJ, et al. Chronological evolution of $\operatorname{IgM}$, IgA, IgG and neutralisation antibodies after infection with SARS-associated coronavirus. Clin Microbiol Infect, 2004,10(12):1062-1066

16 Woo PC, Lau SK, Wong BH, et al. Differential sensitivities of severe acute respiratory syndrome (SARS) coronavirus spike polypeptide enzyme-linked immunosorbent assay (ELISA) and SARS coronavirus nucleocapsid protein ELISA for serodiagnosis of SARS coronavirus pneumonia. J Clin Microbiol, 2005,43(7):3054-3058

17 Woo PC, Lau SK, Wong BH, et al. Longitudinal profile of immunoglobulin $\mathrm{G}$ (IgG), IgM, and IgA antibodies against the severe acute respiratory syndrome (SARS) coronavirus nucleocapsid protein in patients with pneumonia due to the SARS coronavirus. Clin Diagn Lab Immunol, 2004,11(4):665-668

18 Kroll MH. Evaluating interference caused by lipemia. Clin Chem, 2004,50(11):1968-1969

(Received Jun. 30, 2020; accepted Jan. 20, 2021) 